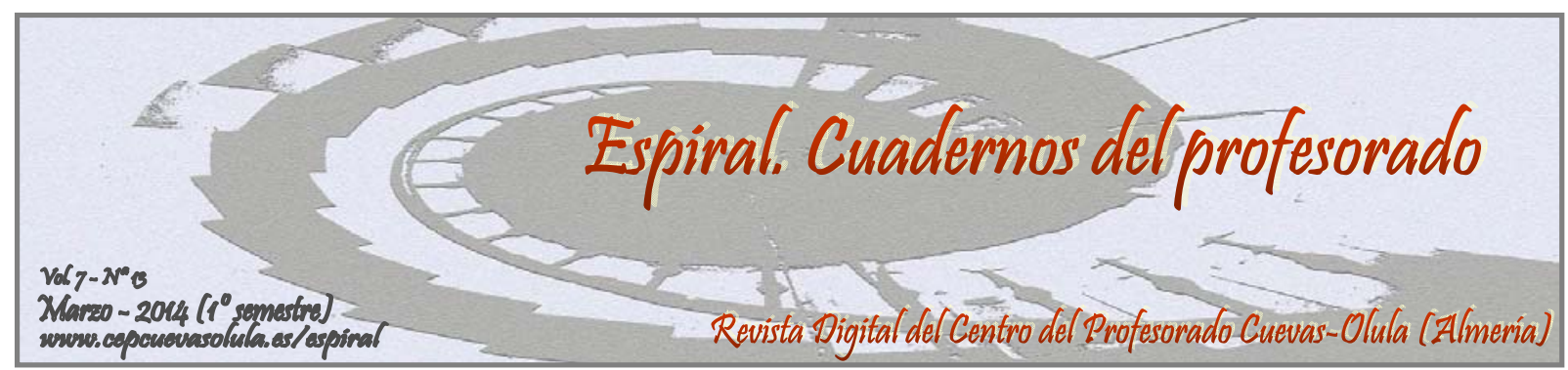

\title{
PROPUESTA DIDÁCTICA PARA EVITAR LAS PRINCIPALES LESIONES EN ESCALADORES
}

\section{DIDACTIC PROPOSAL TO PREVENT THE MAIN INJURES IN CLIMBERS}

\section{Antonio Baena-Extremera ${ }^{(1)}$ y Antonio Granero-Gallegos ${ }^{(2)}$}

(1) Facultad de Ciencias del Deporte, Universidad de Murcia, España

(2) Centro del Profesorado Cuevas-Olula, Consejería de Educación, Cultura y Deporte de la Junta de Andalucía, España

RESUMEN: El objetivo del presente trabajo es doble: por un lado estudiar, a través de una revisión bibliográfica, las lesiones musculares, articulares y tendinosas más comunes en aquellos sujetos que practican escalada, bien en forma de competición o bien en forma de clases educativas. El segundo objetivo es establecer unas propuestas didácticas para mejorar la práctica de este deporte y reducir el número de lesiones producidas en nuestros alumnos. Posteriormente, se establecen unas conclusiones al trabajo.

Palabras clave: lesión, poleas, didáctica, escalada.

ABSTRACT: The aim of this paper is double: first study, through a literature review, muscle injuries, joint and tendon more common in those who practice climbing or in the form of competition or in the form of educational classes. The second objective is to establish educational proposals to improve the practice of the sport and reduce the number of injuries in our students. Later, we suggest some conclusions to work.

Key words: injury, pulleys, eaching, climbing.

Baena-Extremea, A. y Granero-Gallegos, A. (2014). Propuesta didáctica para evitar las principales lesiones en escaladores. Espiral. Cuadernos del Profesorado, 7(13), 44-50. Disponible en: http://www.cepcuevasolula.es/espiral.

Fecha de recepción: 03/12/2013

Fecha de aceptación: 22/02/2014
Enviar correspondencia a: abaenaextrem@um.es

\section{1.- INTRODUCCIÓN}

La escalada es una disciplina que está en auge en todo el mundo, hasta el punto en el que ya se trabaja en los centros educativos con alumnado a partir de 6 años de edad. Este boom tan importante ha supuesto que aumente también la investigación sobre esta materia, enfocada hacia múltiples perspectivas, entre las que se encuentran: la metodología de enseñanza de las técnicas, las lesiones deportivas y su prevención, el entrenamiento y el rendimiento, etc.

Los deportistas que practican escalada dependen, predominantemente, de la fuerza distal y de las extremidades superiores, utilizando cualquiera de las cuatro técnicas de agarre en función del terreno descritas por Jebson y Steyers (1997). Estas cuatro técnicas de agarre tienen como objetivo transmitir fuerzas extremadamente altas a través de los tejidos de los tendones, músculos, manos y 
antebrazos, conllevando a menudo, una gran variedad de posibles lesiones tanto agudas como crónicas.

Durante la práctica y entrenamiento de esta disciplina deportiva, los escaladores tienen que realizar gran cantidad de movimientos y ejercicios isométricos para poder soportar su peso corporal produciéndose, en ocasiones, lesiones crónicas por sobrecarga en los músculos y elementos articulares.

Investigaciones como la realizada por Backe, Ericson, Janson, y Timpka (2009), recogen algunas de las principales lesiones producidas en los deportistas de escalada por un sobreuso y sobreesfuerzo, tales como tendinitis, síndrome del túnel carpiano y fracturas por estrés, especialmente en dedos y muñecas, representando el $75-90 \%$ de las lesiones de escalada (Schöffl \& Winkelmann, 1999; Patrick, 2001; Martinoli, Bianchi, \& Cotton, 2005; Jones, Asghar, \& Llewellyn, 2007). Igualmente, otra de las lesiones más padecidas por estos deportistas es la ruptura de la polea A2 del dedo anular; ésta ha sido descrita como particularmente frecuente entre los deportistas de élite (Rohrbough, Mudge, \& Schilling, 2000; Schöffl, Hochholzer, Winkelmann, \& Strecker, 2003; Schöffl \& Küpper, 2006).

Como vemos, la escalada es una disciplina en la que la mayoría de los deportistas suelen tener alguna lesión a lo largo de su vida deportiva. Por ello, es fundamental aplicar ciertas directrices didácticas en su trabajo, entrenamiento o enseñanza, con el fin de evitar que estas lesiones se produzcan de manera puntual o de forma crónica.

\section{2.- CARACTERÍSTICAS ANTROPOMÉTRICAS DE LOS ESCALADORES Y PRINCIPALES LESIONES}

En relación al estereotipo del deportista de escalada, gran parte de las lesiones pueden deberse a que éstos sujetos no cumplan en gran medida, con el perfil antropométrico idóneo para esta disciplina. Por ejemplo, un perfil de tipo endomorfo supone que el escalador deba ser capaz de aguantar con sus músculos y articulaciones, un peso mayor que otra persona que presente un perfil de tipo ectomorfo. Esto conlleva, por supuesto, posibles sobrecargas tanto a nivel muscular, como articular.

Los estudios han destacado que el perfil antropométrico ideal de estos deportistas estaba descrito por un atleta de pequeña estatura y sobre todo con un bajo porcentaje de grasa y masa corporal (Sheel, 2004; Giles, Rhodes, \& Taunton, 2006) siendo, según Watts (2004), de tipo mesomórfico. De hecho, existen evidencias científicas de una asociación entre la reducción en el porcentaje de grasa corporal y la mejora de rendimiento en la escalada (Sheel, 2004; Caine, DiFiori, \& Maffulli, 2006; Giles et al. 2006).

Teniendo en cuenta lo expuesto, se ha llevado a cabo una revisión de algunos de los trabajos más importantes en antropometría del escalador, con el objetivo de verificar a partir de estos estudios, los perfiles idóneos.

En relación a los datos de la tabla 1, otro trabajo que se puede incluir en ella es el realizado por Backe et al. (2009), quienes encontraron que un mayor IMC se relacionó con un aumento del riesgo de sufrir lesiones en los escaladores que practicaban boulder. Por ello, para evitar posibles lesiones, lo ideal sería que los deportistas se situaran en un perfil aproximado al escalador de élite.

\section{Lesiones en las manos}

En este deporte, las manos se utilizan como herramientas para el ascenso y muchas de las fuerzas externas aplicadas sobre los dedos se distribuyen a través de la muñeca, el codo y el hombro (Hass \& Meyers, 1995; Quaine, Martin, \& Blanchi, 1997). Por ello, una de las principales lesiones producidas en los escaladores se localiza en las manos, debido al esfuerzo físico al que someten, tanto a los músculos de las mismas, como a las articulaciones. Las lesiones aparecen, entre otros motivos, por el tipo de agarre utilizado en la roca (de pinza, invertido, monodedo, bidedo, etc.), ya que cada agarre somete a diferentes partes de la mano a fuerzas extremas. Por ejemplo, un agarre de monodedo 
supone que el escalador deberá aguantar, si fuera preciso, hasta todo su peso corporal con un solo dedo. En él, la articulaciones se flexionan a $90^{\circ}$ y la interfalángica distal se extiende o hiperextensa completamente (Kubiak, Klugman, \& Bosco, 2006). Este tipo de agarre comúnmente conlleva asociado la rotura de poleas articulares. Es imaginable, por tanto, la tensión y el estrés que sufrirá, tanto a nivel muscular como articular.

Tabla 1.- Investigaciones sobre perfiles antropométricos en escaladores.

\begin{tabular}{|c|c|c|c|c|c|c|c|}
\hline Autores & $\begin{array}{c}\mathrm{N}^{\mathrm{o}} \text { sujetos } \\
\text { (hombre/mujer) }\end{array}$ & $\begin{array}{c}\text { Nivel (escala } \\
\text { francesa) }\end{array}$ & $\begin{array}{l}\text { Altura } \\
(\mathrm{m})\end{array}$ & $\begin{array}{c}\text { Peso } \\
(\mathrm{kg})\end{array}$ & IMC & $\begin{array}{c}\text { Grasa } \\
\% \\
\end{array}$ & $\begin{array}{c}\sum 7 \mathrm{SF}^{\mathrm{a}} \\
(\mathrm{mm})\end{array}$ \\
\hline Watts et al., 1993 & $21(\mathrm{H})$ & $8 b$ & $\begin{array}{c}1.778 \\
(0.065)\end{array}$ & $\begin{array}{l}66.6 \\
(5.5\end{array}$ & & $\begin{array}{c}4.7 \\
(1.3)\end{array}$ & $\begin{array}{l}37.8 \\
(6.8)\end{array}$ \\
\hline Grant et al., 1996 & $10(\mathrm{H})$ & $6 a$ & $\begin{array}{c}1.789 \\
(0.085)\end{array}$ & $\begin{array}{l}74.5 \\
(9.6)\end{array}$ & & $\begin{array}{l}14.0 \\
(3.7)\end{array}$ & \\
\hline Watts et al., 1996 & $11(\mathrm{H})$ & $8 \mathrm{a}$ & $\begin{array}{c}1.756 \\
(0.089)\end{array}$ & $\begin{array}{l}65.9 \\
(8.6)\end{array}$ & & $\begin{array}{c}5.4 \\
(1.5)\end{array}$ & $\begin{array}{l}40.8 \\
(7.3)\end{array}$ \\
\hline Mermier et al., 1997 & $9(\mathrm{H})$ & $7 a$ & $\begin{array}{c}1.757 \\
(0.056)\end{array}$ & $\begin{array}{l}66.3 \\
(6.4)\end{array}$ & & $\begin{array}{c}6.8 \\
(2.6)\end{array}$ & \\
\hline Booth et al., 1999 & $6(\mathrm{H})$ & $7 b-8 a$ & $\begin{array}{c}1.757 \\
(0.027)\end{array}$ & $\begin{array}{l}62.6 \\
(3.3)\end{array}$ & $\begin{array}{l}20.3 \\
(2.1)\end{array}$ & & \\
\hline Zapf et al., 2001 & $20(\mathrm{H})$ & $8 \mathrm{a}$ & $\begin{array}{c}1.771 \\
(0.044)\end{array}$ & $\begin{array}{l}65.6 \\
(4.9)\end{array}$ & $\begin{array}{l}20.9 \\
(1.1)\end{array}$ & & \\
\hline Watts et al., 2003 & 38 jóvenes (M) & $6 c$ & 54.4 & 40.6 & $\begin{array}{l}17.5 \\
(2.1)\end{array}$ & $\begin{array}{l}12.2 \\
(2.6)\end{array}$ & $\begin{array}{c}56.0 \\
(14.5)\end{array}$ \\
\hline
\end{tabular}

Nota: $\sum 7 \mathrm{SF}^{\mathrm{a}}$ es la suma de siete mediciones de pliegues cutáneos (pecho, subescapular, axilar medio, suprailíaco, abdomen, tríceps, cuádriceps).

En otro tipo de agarre, como el realizado en las grietas, se asocia por lo general, con el dolor en la primera metacarpofalángica (Cutts \& Bollen, 1993). Como vemos, cada tipo de agarre conllevaría posiblemente lesiones diferentes, específicas del tipo de postura.

Trabajos como el de Bollen y Gunson (1990) y Shea, Shea, y Meals (1992) describen un amplio espectro de lesiones en la escalada que afectan también a los tendones flexores distales. Estas lesiones parecen tener una patogenia común y síntomas similares, incluyendo tendinitis o tenosinovitis, así como rotura.

Otra lesión muy común en las manos es la producida por rotura de las poleas, siendo una lesión específica de estos deportistas. Investigadores como Klauser et al. (2002) y Schöffl y Schöffl (2006), manifiestan que entre el $19 \%$ y $26 \%$ de los escaladores investigados en sus trabajos presentaban estas lesiones, afectando más comúnmente, al dedo anular. Según Kubiak et al. (2006), la mayoría de la literatura afirma que la polea A2 es la primera que se rompe, aunque otros autores como Klauser et al. (2002) y Tang y Xie (2001) afirman que suele ser primera la polea A4. Según Bollen y Gunson (1990), el 40\% de los escaladores de élite tienen signos de insuficiencia en la polea A2.

La polea A2 y A4 tienen como función mantener una relación constante con el eje de la articulación, y acortar menos de un $25 \%$ con la flexión de los dedos. En cambio, las poleas A1, A3 y A5 juegan un escaso papel en la prevención de estas lesiones (figura 1). Por ello, se estima que una de las principales lesiones a cuidar en estos deportistas son las producidas en la A2 y A4, ya que son los más importantes desde el punto de vista biomecánico. La pérdida de cualquiera de las poleas -A2 ó A4- puede resultar una pérdida sustancial de movimiento y de fuerza $\mathrm{y}$, por consiguiente, una reducción del nivel de rendimiento del deportista.

Manske y Lesker $(1983,1977)$ realizaron varias investigaciones en las que probaron la fuerza de las poleas de los dedos en diez cadáveres. Llegaron a la conclusión de que la A1 era la polea más fuerte, con un máximo resistencia a la rotura de $80.8 \mathrm{~kg}$. para el dedo medio, seguido por A4 > A2 > A3. Otros investigadores, como Lin, Cooney, Amadio, y An (1990), realizaron un experimento similar con cinco manos frescas de cadáveres; sin embargo, utilizaron un gancho curvado para cargar las poleas de una manera que se simulara más a la actuación de una polea real. Estos investigadores establecieron el siguiente orden de intensidad de la polea: A2 $>$ A4 $>$ A1 $>$ A3. Además, encontraron que las poleas A2 y A4 tenían menos deformidad bajo similares cargas que el resto de poleas. 
Marco, Sharkey, Smith, y Zissimos (1998) llevaron a cabo otra investigación sobre las lesiones de las poleas. Para ello utilizaron 21 dedos de cadáveres colocados en una posición de agarre estándar de escalada. Estos investigadores encontraron que en 14 de los dedos, la rotura de la polea A4 es la primera que se produjo, a continuación la $\mathrm{A} 2$ en 3 dedos y, por último, y de forma simultánea, en un dedo la rotura de la A3 y A4. En este trabajo se llegó a la conclusión de que la A3 no se rompe la primera debido, fundamentalmente, a que sirve para transferir las fuerzas a la A2 y A4. Otra de las conclusiones fue que la rotura se produjo con mucho menos fuerza en el dedo anular que en el resto de dedos. La importancia de estos aspectos expuestos supone que deban ser tenidos muy en cuenta tanto en la práctica como en el entrenamiento de estos sujetos, a nivel preventivo.

Según Rooks (1997), tres cuartas partes de los deportistas de escalada de élite y recreativos sufren alguna de las lesiones nombradas en las extremidades superiores, siendo el sesenta por ciento de estas lesiones en la mano. En general,

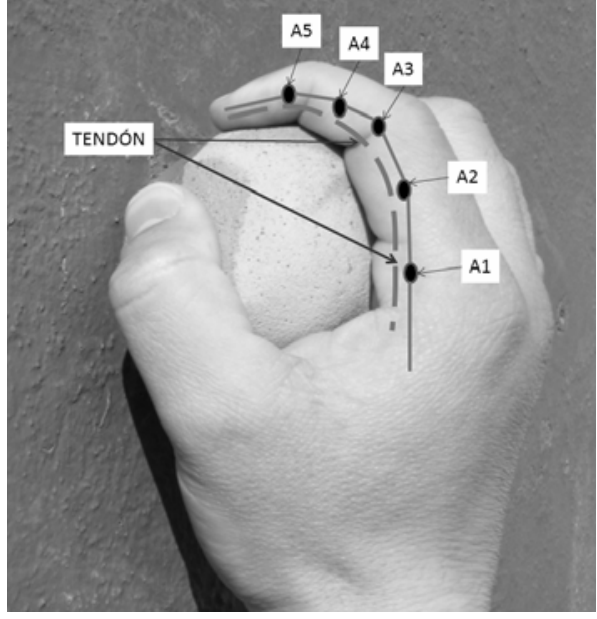

Figura 1.- Agarre de una presa de escalada. Representación gráfica de las poleas A1, A2, A3, A4 y A5 del dedo índice de la mano derecha del escalador. cuando un escalador cree sentir síntomas de lesión en sus manos por uso excesivo de las mismas, comienzan a notar, por la mañana temprano, una mayor rigidez en las manos y un excesivo déficit en la motricidad fina de los dedos. Estas podrían ser las claves de una prevención a tiempo.

\section{Lesiones por caídas}

Kubiak et al. (2006) afirman que algunas de las principales lesiones, sobre todo articulares, son debidas a las caídas durante la escalada. Estas lesiones se producen generalmente en rodillas y tobillos, ya que cuando el deportista cae al vacío, el compañero que "asegura" hace de contrapeso. Esto supone que se tense la cuerda que los une, produciéndose un efecto de péndulo hacia la pared. El escalador que cae debe detener su caída de péndulo hacia la pared mediante el apoyo de sus pies y sus manos, infringiendo, por tanto, una excesiva carga excéntrica en músculos y articulaciones.

Entre los tipos de escalada más lesivos se encuentra la escalada artificial. En ésta el escalador que avanza primero va colocando unos anclajes que retiran el segundo o el último escalador del grupo si van más de dos. Según Kubiak et al. (2006), el mayor peligro es este tipo de escalada está en las caídas, ya que son siempre el doble de la distancia "del escalador" al último anclaje, más el tramo de la cuerda.

Otra de las lesiones por caída se encuentra recogida por Smith y Padgett (1996) en su prestigioso libro "On rope", donde detallan algunos de los síntomas producidos por caídas o por exceso de sujeción del arnés, llamados síndrome compartimental del arnés. Esta lesión es muy común en deportistas que usan un arnés durante horas e incluso días, como por ejemplo alpinistas, escaladores de big wall, espeleólogos o, incluso, los que sufren una caída. Según Hohlrieder, Lutz, Schubert, Eschertzhuber, y Mair (2007), las caídas en los escaladores pueden suponer que el deportista tenga que soportar hasta 6,5 kilonewton $(\mathrm{kN})$ si es una caída importante; por ello, es muy importante la utilización de arneses adecuados y técnicos con el objetivo de evitar estos traumatismos.

\section{3.- PROPUESTA DIDÁCTICA PARA EVITAR LAS POSIBLES LESIONES}

Una vez expuestos los principales casos de lesiones en estos sujetos, vamos a establecer algunas propuestas didácticas que pueden contribuir a evitarlas o a su prevención:

- Para evitar los dolores en la primera metacarpofalángica sería interesante realizar agarres de pinza amplios, en los que el apoyo de los dedos sea perfecto y de ambas manos para poder repartir el 
peso el cuerpo. Para ello deberíamos, como profesores o entrenadores, trabajar con presas que permitan una pinza amplia.

- La rotura de las poleas se produce en el dedo anular con la aplicación de menos fuerza que en el resto. Esto supone que el dedo anular es el más comúnmente involucrado en la rotura de la polea, por lo que debemos organizar el trabajo de forma que los agarres no supongan un exceso de carga en este dedo.

- Una técnica que se puede emplear -y que ya es realizada por muchos escaladores-, es utilizar elementos para tapar la falange proximal y medial; de esta manera se pueden evitar roturas.

- Tras posibles lesiones articulares, y una vez que los deportistas pueden mover libremente la articulación sin dolor consiguiendo cerca del $80 \%$ de la fuerza inicial, se recomienda un mínimo de 6 semanas más hasta llegar a los niveles de escalada máxima. Por ello, es fundamental que a estos pacientes se les instruya para aumentar gradualmente la dificultad y duración de sus entrenamientos durante un período de ocho semanas.

- Schöffl y Kuepper creen que se producen 3.1 lesiones por cada 1000 horas de escalada, entre los 443 competidores en el Campeonato Mundial de 2005 estudiados. Esto nos indica que para reducir las lesiones es fundamental disminuir las horas de trabajo o, por lo menos, la intensidad en las mismas.

- Se ha de proponer apoyos más grandes en el trabajo de boulder, tanto para manos como para pies, aunque la vía de ascenso sea difícil. De esta forma intentamos disminuir la tensión producidas en las manos.

- Muchos de los entrenamientos están diseñados para soportar un gran peso en las extremidades superiores. Este tipo de trabajo no es adecuado para principiantes y, en particular, para los adolescentes cuyos huesos y ligamentos no están plenamente desarrollados (Dennis, Finch, \& Farhart, 2005; Caine et al., 2006; Magra, Caine, \& Maffulli, 2007; Morrison \& Schöffl, 2007).

- Ciertos autores, como Morrison y Schöffl, recomiendan hacer un buen calentamiento y colocar cinta adhesiva para prevenir las lesiones en las articulaciones evitando roturas (Bollen, 1990; Bollen \& Gunson, 1990; Rooks, 1997).

- Siguiendo a Dennis et al. (2005), para evitar lesiones sería interesante seguir una planificación en la que se alterne trabajo y descanso de articulaciones y grupos musculares de forma equilibrada.

- Otra de las aportaciones importantes, es conseguir que los alumnos aprendan a detectar los síntomas iniciales de las lesiones, como la rigidez en la articulación.

- Para evitar las lesiones por caída, se aconseja realizar una escalada en top-rope. De esta forma, el escalador caería entre 1 y 3 metros, evitando el efecto péndulo hacia la pared y disminuyendo la posibilidad de producir lesiones.

- Otra recomendación para la escalada clásica sería introducir un mayor número de seguros, de manera que se disminuyera la distancia entre los mismos y, por tanto, la distancia de caída, si se produjese.

- Para evitar las posibles lesiones por síndrome compartimental se aconsejaría utilizar un arnés con mayor acolchado en las piernas, así como introducir descanso con una mayor frecuencia.

- Otra de las opciones para evitar el daño producido por el arnés debido a una fuerte caída sería utilizar un aseguramiento dinámico, para que la parada del sujeto que cae no sea de forma brusca.

\section{4.- DISCUSIÓN Y CONCLUSIONES}

La mayoría de las lesiones producidas en la escalada son ocasionadas en las extremidades superiores, tal como recogen autores como Backe et al. (2009), Jones et al. (2007), Josephsen et al. (2007), Martinoli et al. (2005), y Patrick (2001), entre otros. Aunque es de destacar que en el trabajo de Josephsen et al. (2007), se concluye que en el caso del boulder, una de las principales causas de lesiones en extremidades inferiores se deben a las caídas del escalador. 
Schöffl et al. (2006), realizaron un trabajo en el que recogieron la localización de las principales lesiones en la escalada, siendo el $41 \%$ en los dedos, un $13 \%$ los codos, un $5 \%$ en los hombros y un $4 \%$ en las rodillas. Lo que está claro es que los dedos son los segmentos corporales que se lesionan con mayor frecuencia, coincidiendo con las aportaciones de Gerdes, Hafner, y Aldag (2006), Rohrbough et al. (2000), y Wright, Royle, y Marshall (2001).

En la literatura hemos encontrado ya algún trabajo que estudia las medidas preventivas para evitar estas lesiones, como por ejemplo el trabajo de Josephsen et al. (2007). A pesar de ello, estos autores manifiestan la ineficacia de las medidas tomadas comúnmente para reducir el número total de lesiones. Con este trabajo, se pretende aportar nuevas directrices didácticas, tanto para la enseñanza como para el entrenamiento, con vista a mejorar las lesiones nombradas en el texto. Por tanto, una futura línea de investigación podría ir enfocada a comprobar el éxito o fracaso de las medidas aquí aportadas, y discutir con los trabajos ya existentes.

\section{5.- REFERENCIAS}

Backe, S., Ericson, L., Janson, S., \& Timpka, T. (2009). Rock climbing injury rates and associated risk factors in a general climbing population. Scandinavian Journal of Medicine and Science of Sports, 19, 850-856

Bollen S. R. (1990). Injury to the A2 pulley in rock climbers. Journal of Hand Surgey, 15B, 268-270.

Bollen S. R. \& Gunson C. K. (1990). Hand Injuries in competition climbers. British Journal of Sport Medicine, 24, 16-18.

Booth, J., Marino, F., Hill, C., \& Gwinn, T. (1999). Energy cost of sport rock climbing in elite performers. British Journal of Sports Medicine, 33, 14-18.

Caine, D., DiFiori, J., \& Maffulli, N. (2006). Physical injuries in children's and youth sports: reasons for concern? British Journal of Sports Medicine, 40, 749-760.

Cutts, A. \& Bollen, S. R. (1993). Grip strength and endurance in rock climbers. Proceedings of the Institution of Mechanical Engineers, 207(2), 87-92.

Dennis, R. J., Finch, C. F., \& Farhart, P. J. (2005). Is bowling workload a risk factor for injury to Australian junior cricket fast bowlers? British Journal of Sports Medicine, 39, 843-846.

Gerdes, E. M., Hafner, J. W., \& Aldag, J. C. (2006). Injury patterns and safety practices of rock climbers. Journal of Traumatology, 61(6), 1517-1525.

Giles, L. V., Rhodes, E. C., \& Taunton, J. E. (2006). The physiology of rock climbing. Sports Medicine, 36(6), 529-545.

Grant, S., Hynes, V., Whittaker, A., \& Aichison, T. (1996). Anthropometric, strength, endurance and flexibility characteristics of elite and recreational climbers. Journal of Sports Science, 14, 301-309.

Hass, J. C. \& Meyers, M. C. (1995). Rock climbing injuries. Sports Medicine, 20(3), 199-205.

Hohlrieder, M., Lutz, M., Schubert, H., Eschertzhuber, S., \& Mair, P. (2007). Pattern of Injury After RockClimbing Falls Is Not Determined by Harness Type. Wilderness \& Environment Medicine, 18, 30-35.

Jebson, P. J. L. \& Steyers, C. M. (1997). Hand Injuries in Rock Climbing: Reaching the Right Treatment. Physician Sportsmedicine, 25(5).

Jones, G., Asghar, A., \& Llewellyn, D. J. (2007). The epidemiology of rock climbing injuries. British Journal of Sports Medicine, 7, 1-16.

Josephsen, G., Shinneman, S., Tamayo-Sarver, J., Josephsen, K., Boulware, D. Hunt, M., \& Pham, H. (2007). Injuries in Bouldering: A Prospective Study. Wilderness and Environment Medicine, 18, 271-280.

Klauser, A., Frauscher, F., Bodner, G., Halpern, E., Schocke, M. F., Springer, P., \& Zur Nedden, D. (2002). Finger pulley injuries in extreme rock climbers: Depiction with dynamic US. Radiology, 222(3), 755761.

Kubiak, E. N., Klugman, J. A., \& Bosco, J. A. (2006). Hand Injuries in Rock Climbers. Bulletin of the NYU Hospital for Joint Diseases, 64(3-4), 172-177.

Lin, G. T., Cooney, W. P., Amadio, P. C., \& An, K. N. (1990). Mechanical properties of human pulleys. Journal of Hand Surgery, 15(4), 429-434.

Magra, M., Caine, D., \& Maffulli, N. (2007). A review of epidemiology of paediatric elbow injuries in sports. Sports Medicine, 37(8), 717-735.

Manske, P. R., \& Lesker, P. A. (1977). Strength of human pulleys. Hand, 9(2), 147-152.

Manske, P. R., \& Lesker, P. A. (1983). Palmar aponeurosis pulley. Journal of Hand Surgery, 3, 259-263.

Marco, R. A., Sharkey, N. A., Smith, T. S., \& Zissimos, A. G. (1998). Pathomechanics of closed rupture of the flexor tendon pulleys in rock climbers. Journal of Bone and Joint Surgery-American, 80(7), 1012-1019. 
Martinoli, C., Bianchi, S., \& Cotton, A. (2005). Imaging of rock climbing injuries. Semin Musculoskelet Radiol, 9(4), 334-345.

Mermier, C. M., Robergs, R. A., McMinn, S. M., \& Heyward, V. H. (1997). Energy expenditure and physiological responses during indoor rock climbing. British Journal of Sports Medicine, 31, 224-228.

Morrison A. B. \& Schöffl V. R. (2007). Physiological responses to rock climbing in young climbers. British Journal Sports Medicine, 41, 852-861.

Patrick, P. (2001). Orthopaedic problems in sport climbing. Wilderness \& Environment Medicine, 12, 100-110.

Quaine, F., Martin, L., \& Blanchi, J. P. (1997). The effect of body position and number of supports on wall reaction forces in rock climbing Journal of Applied Biomechanic, 13, 14-23.

Rohrbough, J. T., Mudge, M. K., \& Schilling, R. C. (2000) Overuse injuries in the elite rock climber. Medicine and Science in Sports and Exercise, 32(8), 1369-1372.

Rooks, M. D. (1997). Rock climbing injuries. Sports Medicine, 23, 261-270.

Schöffl, V. R. \& Kuepper, T. (2006). Injuries at the 2005 World Championships in rock climbing. Wilderness \& Environment Medicine, 17(3), 187-190.

Schöffl, V. R. \& Schöffl, I. (2006). Injuries to the finger flexor pulley system in rock climbers: Current concepts. Journal of Hand Surgery, 31(4), 647-654.

Schöffl, V. R., Hochholzer, T., Winkelmann, H. P., \& Strecker, W. (2003). Pulley injuries in rock climbers. Wilderness \& Environment Medicine, 14(2), 94-100.

Schöffl, V. R. \& Winkelman, H. P. (1999). Unfallstatistik an “Indoor-Kletterlagen'. Sportververletz Sportschaden, 13, 14-16.

Shea, K. G., Shea, O. F., \& Meals, R. A. (1992). Manual demands and consequences of rock climbing. Journal of Hand Surgery, 17(2), 200-205.

Sheel, A. W. (2004). Physiology of sport rock climbing. British Journal of Sports Medicine, 38, 355-359.

Smith, B. \& Padgett, A. (1996). On rope. National speleological society: Huntsville, Alabama.

Tang, J. B. \& Xie, R. G. (2001). Effect of A3 pulley and adjacent sheath integrity on tendon excursion and bowstringing. Journal of Hand Surgery, 26(5), 855-861.

Watts, P. B. (2004). Physiology of difficult rock climbing. European Journal of Applied Physiology, 91, 361372.

Watts, P. B. \& Jensen, R. L. (2003). Reliability of peak forces during a finger curl motion common in rock climbing. Measurement in Physical Education and Exercise Science, 7, 263-267.

Watts, P. B., Martin, D. T., \& Durtschi, S. (1993). Anthropometric profiles of elite male and female competitive sport rock climbers. Journal of Sports Science, 11, 113-117.

Watts, P. B., Newbury, V., \& Sulentic, J. (1996). Acute changes in handgrip strength, endurance, and blood lactate with sustained sport rock climbing. Journal of Sports Medicine and Physical Fitness 36, 255 260.

Wright, D. M., Royle, T. J., \& Marshall, T. (2001). Indoor rock climbing: who gets injured? British Journal of Sports Medicine, 35(3), 181-185.

Zapf, J., Fichtl, B., Wielgoss, S., \& Smidth, W. (2001). Macronutrient intake and eating habits of elite rock climbers. Medicine and Science in Sports and Exercise, 33(5), S72

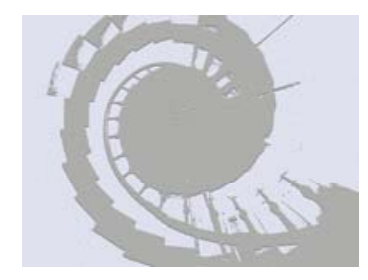

